\title{
Hausarztwechsel oder Kassenwechsel? Ideen zu einem neuen Paradigma
}

\section{Christian Marti ${ }^{a}$}

a Der Autor ist Geschäftsleiter des Ärztenetzwerks Wintimed AG in Winterthur.
* Mit «Hausarztmodell» sind in diesem Artikel ausschliesslich alternative Versicherungsmodelle mit expliziter, vertraglich geregelter Beteiligung der Hausärztinnen gemeint.

** Die Zukunft der Medizin ist weiblich. Die männliche Form ist immer mitgemeint.

- Dans le présent article, le terme de «modèle du médecin de famille» se réfère uniquement aux modèles d'assurance alternatifs qui comprennent la participation explicite des médecins de premie recours et la règlent contractuellement.

Korrespondenz:

Dr. med. Christian Marti

Rosinliweg 44

CH-8400 Winterthur

christian.marti@hin.ch

\section{Einleitung}

Grundversorger und Grundversicherer haben eines gemeinsam: ihre Zielgruppe ist die gesamte Bevölkerung und deren Gesundheit. Es gibt grundversorgerfreundliche Versicherer. Sie fördern im Rahmen vertraglich geregelter Hausarztmodelle* die Entwicklung und Erprobung einer qualitativ hochstehenden und effizienten Grundversorgung inklusive neuer Praxisformen. Andere Versicherungen beuten die gemeinsamen Anstrengungen von Pionierkassen und Ärztenetzen aus, ohne sich selbst daran zu beteiligen. Da Krankenkassen seit Jahren mit einer wachsenden Zahl von Versicherungsprodukten die Wahl der Hausärztin** und anderer Leistungserbringer beeinflussen, stellt sich die Frage: Warum sollen Hausärztinnen nicht ihrerseits die Krankenkassenwahl ihrer Patienten zugunsten von hausarztfreundlichen Versicherungen beeinflussen? Hausärztliche Standesorganisationen verfolgen die Entwicklungen neuer Praxisformen eher passiv oder ambivalent. Liegt die Zukunft der bedrohten Hausarztmedizin in den Händen dieser Standesorganisationen oder in einer Allianz fortschrittlicher Hausärzte mit weitblickenden Versicherungen?

\section{Hausarztmedizin in der Krise}

Es gibt immer mehr Ärzte, aber immer weniger Hausärzte. Die Hausarztmedizin steckt in einer Krise. Eine Spezialistenmedizin, die lukrativer und spektakulärer ist als die aufreibende Hausarztmedizin, eine wachsende Flut von Vorschriften, immer mehr Abstriche an den Kompetenzen, eine immer noch kümmerliche Existenz der Hausarztmedizin in Lehre und Forschung und nicht zuletzt das dauernde Gejammer der Hausärzte selbst, sie alle rauben der Hausarztmedizin jede Anziehungskraft und Faszination. Kein Wunder, wählt nur noch eine von zehn Personen mit abgeschlossenem Medizinstudium die Fachrichtung Allgemeinmedizin.

\section{Krankenkassen als Förderer der Hausarztmedizin}

Trotz unterschiedlicher Interessenlage haben Grundversorger und Grundversicherer doch eine wesentliche Gemeinsamkeit. Ihre Zielgruppe ist

\section{Changer de médecin de famille ou de caisse-maladie?}

Les médecins de premier recours et les assureurs de base ont un point commun: ils ont pour cible l'ensemble de la population et sa santé. Il existe des assureurs favorables aux médecins précités. Dans le cadre de modèles «médecin de famille»" ils encouragent le développement et l'expérimentation d'une médecine de premier recours efficace et de haute qualité, ainsi que de nouvelles formes de cabinets médicaux. D'autres assureurs exploitent, sans y participer du tout, les efforts déployés en commun par des caisses pionnières et des réseaux de médecins. Vu que les caisses-maladie influencent depuis des années le choix du médecin de famille et d'autres prestataires de soins en proposant un nombre croissant de produits d'assurance, on peut se demander pourquoi les médecins de famille $n$ 'influenceraient pas leurs patients quant aux choix d'une caisse favorisant la médecine de premier recours. Les organisations professionnelles des praticiens de premier recours suivent l'évolution des nouvelles formes de cabinet de manière plutôt passive ou ambivalente. Le futur de la médecine de famille, discipline en danger, réside-t-il dans ces organisations ou plutôt dans une alliance de médecins progressistes et d'assureurs clairvoyants?

die gesamte Bevölkerung, also Alte und Junge, Männlein und Weiblein, Gesunde und Kranke und zwar alle Kranken. Spezialisten dagegen sind Ansprechpartner für viel kleinere Zielgruppen, die Kardiologen zum Beispiel für Patienten mit Kreislaufkrankheiten, die Onkologen für Tumorpatienten. Als Spezialisten verfolgen sie in erster Linie die Nutzenmaximierung für ihre eigene, kleine Zielgruppe - ohne Rücksicht auf Patien- 
ten ausserhalb ihres Fachbereiches. Generalisten und Grundversicherer hingegen als Ansprechpartner der gesamten Bevölkerung haben eher das Gesamtsystem mit allen Versicherten im Auge.

Seit 15 Jahren wählt in der Schweiz ein wachsender Anteil der Bevölkerung Hausarzt- und HMO-Modelle. Beiden gemeinsam ist, dass Patientinnen und Patienten für die Erstbeurteilung bei gesundheitlichen Problemen eine Grundversorgerin aufsuchen. Eng damit verknüpft hat sich eine Vielfalt von Ärztenetzen entwickelt, mit und ohne Einbezug von Spezialisten oder andern Leistungserbringern, mit unterschiedlicher oder fehlender Budgetverantwortung, mit gelegentlichen oder wöchentlichen Qualitätszirkeln, mit oder ohne externe Überprüfung verschiedener Qualitäten (Zertifizierung). In vielen dieser Ärztenetze beschäftigt man sich intensiv mit neuen Rollen der Hausärztin und mit neuen Praxisformen. Gleichzeitig entwickeln sich allmählich professionelle Netzstrukturen, die dem fachlichen und ökonomischen Support der einzelnen Praxen dienen. Diese Entwicklung haben bis jetzt mehrere grundversorgerfreundliche Krankenversicherungen im Rahmen von vertraglich geregelten, «echten» Hausarztmodellen gefördert und in Form von Steuerungsentschädigungen und/oder Erfolgsbeteiligungen auch finanziell mitgetragen.

\section{Krankenkassen als Ausbeuter der Hausarztmedizin}

Von vielen Verbesserungen oder Einsparungen, welche in den Ärztenetzen erarbeitet und umgesetzt werden, profitieren alle Patientinnen, unabhängig davon, ob sie konventionell versichert sind oder ob sie ein Hausarzt- oder HMOModell gewählt haben, also auch unabhängig davon, ob sie eine Versicherung gewählt haben, welche überhaupt ein Hausarzt- und HMO-Modell anbietet oder nicht.

Beispiel: Wenn Netze aufgrund eines evidenzbasierten Vergleichs aller kassenpflichtigen Säureblocker zum Schluss kommen, dass diese für die ganz grosse Mehrzahl der Patientinnen im Praxisalltag medizinisch gleichwertig sind und sich gemeinsam auf ein bestimmtes günstiges Generikum als Säureblocker der ersten Wahl einigen, dann profitieren davon automatisch alle Kassen. Also auch all jene, die, aus welchen Gründen auch immer, noch nie Zeit und Geld in den Betrieb eines Hausarztmodells investiert haben. Wir nennen sie Trittbrettfahrer. Andere Profiteure unter den Versicherungen bieten Pseudohausarztmodelle aufgrund von Hausarztlisten an, ohne die Hausärzte einzubeziehen, und belästigen sie gleichzeitig mit administrativem Mehraufwand für Überweisungsbestätigungen und dergleichen.
Wir nennen sie die Etikettenschwindler. Und schliesslich gibt es auch jene Versicherungen, die verschiedene Netze betreffend Abgeltungen in den gleichen Topf werfen wollen, unabhängig davon, ob sich diese einmal wöchentlich oder einmal monatlich zu Qualitätszirkeln bzw. Steuerungssitzungen treffen, unbesehen davon, wie viele erfolgreiche Innovationen ein Netz entwikkelt, und ohne Rücksicht darauf, ob sich ein Netz regelmässig einer externen Qualitätsbeurteilung (Zertifizierung) unterzieht. Es fragt sich, wie lange noch engagierte Kassen und Ärztenetze diese Ausbeutung durch andere Kassen dulden können und wollen.

\section{Alle Patientinnen gleich behandeln?}

Es ist sehr einfach, Kostenunterschiede zwischen konventionell Versicherten und solchen in alternativen Versicherungsmodellen $\mathrm{zu}$ erfassen. Wesentlich schwieriger ist es, diese zu interpretieren. So oder so stehen für die Versicherungen diese Kostendifferenzen im Zentrum der Vergleiche und der Verhandlungen mit Hausarztnetzen. Je grösser diese Kostendifferenz, um so attraktiver ist eine Ärztegruppe als Vertragspartner. Für die einzelne Hausärztin stellt sich darum im Praxisalltag die Frage, in welcher Hinsicht sie Patientinnen im Hausarztmodell anders behandeln will als konventionell versicherte. Soll sie für die einen konsequent teure, noch nicht generikafähige Säureblocker einsetzen und für die andern das günstigste, medizinische aber gleichwertige Omeprazol-Generikum? Soll sie die einen mit Bagatellverletzungen der Gelenke bewusst grosszügig und bereits bei der ersten Konsultation zu MRI-Untersuchungen schicken, wenn der Patient es wünscht, und die andern möglichst gezielt nach Befund und Verlauf? Soll sie die einen absichtlich zu jenem Spezialisten schicken, der bekannt dafür ist, den Abklärungsauftrag grosszügig auszulegen und den Tarif noch grosszügiger auszureizen?

Betreffend medizinische Qualität wollen die Hausärztinnen alle ihre Patientinnen ohne Abstriche und gleich gut behandeln - unabhängig vom Versicherungsstatus, also auch unabhängig davon, ob jemand ein Hausarztmodell gewählt hat oder nicht. Im Sinne der organisatorischen oder der Servicequalität können professionelle Netze den Versicherten, welche das Hausarztmodell gewählt haben, mit etwas Phantasie und Geschick eine Vielfalt von gezielten Vergünstigungen gewähren, zum Beispiel bei Impfaktionen, für Taxifahrten, bei ausgewählten Apotheken und Optikern. Sie sollten jede Gelegenheit nutzen, jene Versicherten mit kleinen Zusatzleistungen ein bisschen zu verwöhnen, welche ihnen 
mit der Wahl des Hausarztmodells ein spezielles Vertrauen ausdrücken. Dies erfordert aber, dass auch die Praxis laufend genau unterscheidet zwischen konventionell Versicherten und Versicherten in verbindlich geregelten Hausarztmodellen. Die Hausärztin, die alle ihre Patienten nicht nur medizinisch, sondern auch organisatorisch gleich behandeln will, könnte entweder nur noch konventionell Versicherte betreuen oder nur solche, die ein echtes Hausarztmodell einer Partnerkasse ihres Netzes gewählt haben.

\section{Standespolitische Ohnmacht}

Es gibt zwei grosse hausärztliche Standesorganisationen in der Schweiz, das Kollegium für Hausarztmedizin und die Schweizerische Gesellschaft für Allgemeinmedizin. Als Spiegelbild ihrer Mitglieder stehen sie diesen Entwicklungen eher ambivalent und passiv gegenüber. «Es braucht neue Praxismodelle», zitiert die Neue Zürcher Zeitung den Präsidenten einer dieser Organisationen [1]. Obwohl aber beide Organisationen jährlich mehrtägige Fortbildungsveranstaltungen durchführen, an denen sich Hunderte von Hausärztinnen aus allen Landesgegenden beteiligen, ist nicht ein einziges Referat, nicht ein einziger Workshop der diesjährigen Grossveranstaltungen der Frage nach neuen, attraktiven und zukunftsfähigen Praxisformen gewidmet [2, 3].

Bei jeder Tarifrevision bleiben die anstössigen Einkommensdifferenzen zwischen Grundversorger- und Spezialistenmedizin unangetastet. Politik und Öffentlichkeit signalisieren immer wieder und deutlich, dass dies ein innerärztliches Problem sei. Wo sind die Erfolge der hausärztlichen Standesorganisationen im Tarifbasar? Was braucht es noch, dass sie sich offen dem unvermeidlichen innerärztlichen Verteilungskampf um die begrenzten finanziellen Ressourcen stellen?

\section{Hausarztwechsel oder Kassenwechsel?}

Heute arbeiten viele Hausärztinnen und Hausärzte an der Grenze ihrer psychischen und physischen Leistungsfähigkeit. Entlastung durch Nachwuchs ist nicht in Sicht. «Sie suchen eine Hausärztin? Bei welcher Krankenkasse sind Sie versichert? Tut uns leid, wir sind so ausgelastet, dass wir nur noch neue Patienten von solchen
Versicherungen nehmen, die mit uns zusammen ein echtes Hausarztmodell anbieten.»

Was wäre denn, wenn die Mitglieder professioneller Ärztenetze ihre Dienste überhaupt nur noch Mitgliedern jener Partnerkassen anbieten würden, die den Hausärzten administrative Erleichterungen und eine bessere Abgeltung offerieren - als Gegenleistung für konsequente und messbare Verbesserungen der Patientenversorgung? Gelegenheit dazu bietet unser fragmentiertes Gesundheitswesen in Hülle und Fülle. «Sehr geehrter Herr Muster, zu meiner Entlastung werde ich ab 1. Januar nur noch Versicherte von Kassen betreuen, die zusammen mit unserer Ärztegruppe ein echtes Hausarztmodell anbieten. Das sind nämlich jene Versicherungen, die sich für eine zukunftsfähige und attraktive Hausarztmedizin einsetzen. Derzeit sind dies folgende Versicherungen: ... Bitte prüfen Sie anhand dieser Liste rechtzeitig, ob sich für Sie eher die Frage eines Kassen- oder eines Hausarztwechsels stellt. Für weitere Auskünfte stehe ich Ihnen gerne zur Verfügung.»

\section{Paradigmenwechsel?}

Hausarztwechsel oder Kassenwechsel? Mit einer wachsenden Zahl von Versicherungsprodukten, den sogenannten alternativen Versicherungsmodellen, beeinflussen Krankenkassen seit Jahren die Wahl der Hausärztin und anderer Leistungserbringer. Warum sollen Hausärztinnen nicht ihrerseits die Krankenkassenwahl ihrer Patienten zugunsten von weitblickenden Versicherungen beeinflussen, die eine zukunftsfähige Hausarztmedizin fördern? Mit denen sie gemeinsam die strukturellen Probleme für eine patientenorientierte Grundversorgung wirksam angehen können? Liegt die Zukunft der bedrohten Hausarztmedizin vielleicht in einer Allianz fortschrittlicher Hausärzte mit weitblickenden Versicherungen?

\footnotetext{
Literatur

1 Neue Zürcher Zeitung vom 10.4.2007: «Es braucht neue Praxismodelle».

2 www.sgam.ch/pdf/sgam07_vorproDef.pdf.

3 www.congress-info.ch/khm2007.
} 